\title{
THE ASSAY OF GALACTOKINASE AND GALACTOSE-1-PHOSPHATE URIDYL TRANSFERASE ACTIVITY IN HUMAN ERYTHROCYTES
}

\section{A Presumed Test for Heterozygous Carriers of the Gatactosemic Defect*}

BY ARTHUR ROBINSON, M.D.

(From the Department of Biophysics and the Department of Pediatrics, University of Colorado Medical Center, Denver)

(Received for publication, May 6, 1963)

Galactosemia, an inborn error of metabolism, is transmitted as an autosomal, recessive gene (1-3), and is chemically characterized by a defect in the enzyme Gal-1-P uridyl transferase (4). This results in the inability to convert galactose to glucose, and in the consequent accumulation of Gal-1-P in the tissues (5). Possibly as a toxic manifestation of this accumulation (6), hepatosplenomegaly, cataracts, mental retardation, convulsions, jaundice, gastrointestinal disturbances, and death may supervene in affected infants in whom galactose has not been removed from the diet.

The metabolic pathways involved in galactose metabolism follow (7-10):1

(a) Gal + ATP $\stackrel{\text { galactokinase }}{\longrightarrow}$ Gal-1-P + ADP

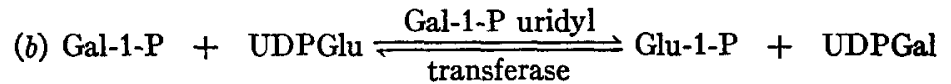

(c) UDPGal $\stackrel{\text { UDPGal-4-epimerase }}{\rightleftharpoons}$ UDPGlu

(d) Glu-1-P + UTP $\stackrel{\text { UDPGlu pyrophosphorylase }}{\rightleftharpoons}$ UDPGlu + PP

(e) Gal-1-P + UTP $\stackrel{\text { UDPGal pyrophosphorylase }}{=}$ UDPGal + PP

* From the Department of Biophysics (Contribution No. 208) and from the Department of Pediatrics, University of Colorado Medical Center, Denver. This work was aided by a grant from The National Foundation and by a training grant, T1 GM 781-04, from the National Institute of General Medical Sciences.

1 Symbols: Gal, galactose; ATP, adenosine triphosphate; Gal-1-P, galactose-1-phosphate; Glu-1-P, glucose-1-phosphate; UDPGal, uridine diphosphogalactose; UTP, uridine triphosphate; PP, pyrophosphate. 
The first three of these reactions are known to occur in red cells, but reaction $e$ which has been demonstrated in other tissues, has not been shown in red cells (10).

From the above reactions it is clear that a block in reaction $b$ unaccompanied by a large forward reaction in step $e$ would result in an accumulation of Gal-1-P.

Persons with galactosemia then may be characterized as Gal-1-P uridyl transferase mutants comparable to the galactose mutants of Escherichia coli. In E. coli, however, mutants have been found involving blocks in reactions $a$ and $c$ as well as $b$ (11). Similar blocks have not been reported in mammals, possibly because they have not been recognized. However, one patient has been reported in whom a galactokinase block has been postulated (12), and an epimerase block has been described in $\mathrm{L}$ cells (13).

It seemed of interest, therefore, to adapt a method previously utilized by Morse (14) for the investigation of galactose mutants of $E$. coli, to human erythrocytes for the purpose of studying galactokinase and Gal-1-P uridyl transferase in persons varying in genotype with respect to galactose utilization. The method consists of incubating erythrocytes in the presence of galactose-1$\mathrm{C}^{14}$ and analyzing the distribution of the resulting $\mathrm{C}^{14}$-labeled compounds.

\section{Method}

1. The red blood cells in $0.75 \mathrm{cc}$ of heparinized, freshly drawn blood are washed in the cold with a buffer $\left(10^{-8} \mathrm{M}\right.$ tris in $0.14 \mathrm{M}$ sodium chloride containing $50 \mathrm{mg}$ per cent D-glucose). Duplicate samples are prepared from each patient. Blood from an individual known to be normal is always run in parallel with a series of test bloods.

2. The washed red blood cells are reconstituted to their original volume by the addition of cold buffer. This suspension of erythrocytes may be refrigerated overnight.

3. To the suspension is added UDPGal $(0.05 \mathrm{mg})$ to act as a carrier for the purpose of possibly trapping any radioactive UDPGal produced enzymatically, which might otherwise be lost either by subsequent enzymatic transformation or by non-specific processes like adsorption to glass; $;^{2}$ and 0.25 microcurie D-galactose-1- $\mathrm{C}^{14}$. $^{3}$ The tubes are placed in a water bath at $37^{\circ} \mathrm{C}$ for exactly 2 minutes, then removed and plunged into an ice bath.

4. The tubes are centrifuged in the cold for 2 minutes at $1500 \mathrm{RPM}$ in an International clinical centrifuge; the supernatant liquid is removed and the red blood cells are washed in the same cold buffer to which $50 \mathrm{mg}$ per cent of $\mathrm{D}$-galactose has been added. The washed cells are restored to original volume $(0.75 \mathrm{cc})$ with cold, distilled water to induce hemolysis. The tube is then immersed in boiling water for 90 seconds to inactivate enzymes and precipitate proteins, and finally is centrifuged until the supernatant liquid is clear.

5. $0.1 \mathrm{ml}$ of the clear supernatant liquid is applied to Whatman No. 4 chromatograph paper ( 1 inch wide) which has been previously washed in Leloir's ethanol-acetate solvent (1 M sodium acetate $\mathrm{pH} 7.5,30 \mathrm{cc} ; 95$ per cent ethanol, $75 \mathrm{cc}$ ), and ascending paper chromatography is carried out in the same solvent. The dried papers are cut into strips $5 \mathrm{~mm}$ wide, using a semiautomatic paper-cutting device. ${ }^{4}$ The strips are placed, in order, into consecutively numbered bottles of a scintillation counter ${ }^{5}$ Each bottle contains $10 \mathrm{cc}$ of a mixture of PPO,

\footnotetext{
${ }^{2}$ Uridine 5 -diphosphogalactose sodium, Sigma Chemical Company, St. Louis.

'Specific activity $8.0 \mathrm{mc} / \mathrm{mmole}$, Volk Radio-Chemical Company, Chicago.

4 "Slide-n-cut," Safety Cutter Company, Menlo Park, California.

${ }^{5}$ Tri-carb liquid scintillation spectrometer, Packard Instrument Company, Inc., La Grange, Illinois.
} 
POPOP, and toluene (PPO, $2 \mathrm{gm}$; POPOP, $50 \mathrm{mg}$; toluene, $500 \mathrm{cc}$ ).6 Graphs are then constructed of the location of radioactivity on the chromatograph strips. The sites of radioactivity conform to the R.F.'s ${ }^{7}$ for Gal (0.65), Gal-1-P (0.32), and UDPGal (0.18) originally determined by Leloir (7) for this solvent and confirmed by us.

6. The ratio of the amount of radioactivity in the Gal-1-P position to that in the UDPGal position $\left(\frac{\mathrm{Gal}^{*}-1-\mathrm{P}}{\mathrm{UDPGal}^{*}} \times 100\right)$ is computed as a measure of the relative activity of the transferase enzyme and is called the " $R$ " value.

In Figs. 1 to 3 are respectively presented typical radioactivity distributions obtained from the chromatography of $(a)$ a presumably normal subject, $(b)$ a patient with galactosemia, and $(c)$ a heterozygous carrier of the galactosemic defect. The corresponding " $\mathrm{R}$ " values are indicated.

\section{EXPERIMENTAL RESULTS}

1. Results on Presumably Normal Persons from a Random Population.--In order to obtain a baseline for this test, 37 randomly chosen people from the general population were subjected to the procedure. Some of these were anonymous blood donors. The " $R$ " values obtained are presented in Table I and reveal a mean and standard deviation of 80 and 12.4, respectively. In addition, 12 consecutive determinations were carried out during a period of 3 weeks on a single, presumably normal individual. The latter data presented in Table II show a mean and standard deviation which are reasonably close to the respective values obtained on the random population. The data of Table I reveal that only 3 of the 37 individuals tested differ from the mean by 2 standard deviations or more. Of these, 1 was pregnant, and it is conceivable that this condition may affect the test. The other 2 values were obtained on blood samples from unidentified donors. The fact that these 2 values deviated by approximately 3 standard deviations from the mean is not as significant as it might seem, since these particular determinations were carried out during the early period of development of the methodology before the measures needed to insure maximum reliability of the results had been identified. If a new mean were computed without these 3 values, it would be appreciably closer to that found in the repeated determinations on the same person, as listed in Table II. However, for the present all of the data have been retained in order to treat them most conservatively. Further assays on larger groups of presumably normal persons are planned in order to fix more definitely the frequency of occurrence of deviations of various magnitudes in a random population.

2. Results on Patients with Galactosemia. - Three patients with galactosemia were available and have been tested. As would be expected, no demonstrable

${ }^{6}$ PPO, 2,5 diphenyloxazole; POPOP, 1,4 bis-2-(5-phenyloxazolyl)-benzene, Packard Instrument Company, Inc., LaGrange, Illinois; toluene, scintillation grade, Matheson Coleman and Bell, Cincinatti.

7 R.F.: This term is used in chromatography to locate the position of a molecule on the chromatograph paper "relative to the solvent front." 


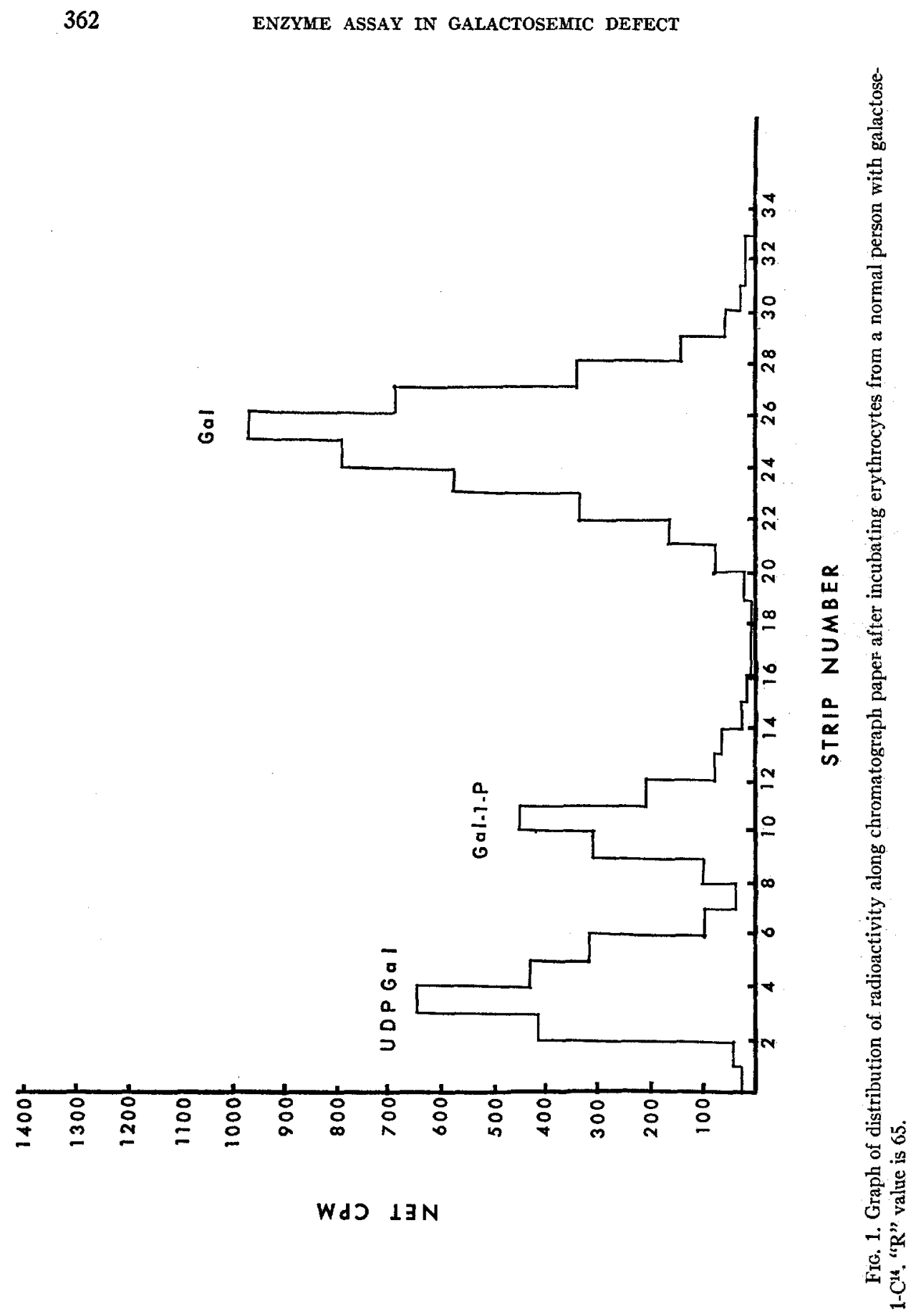




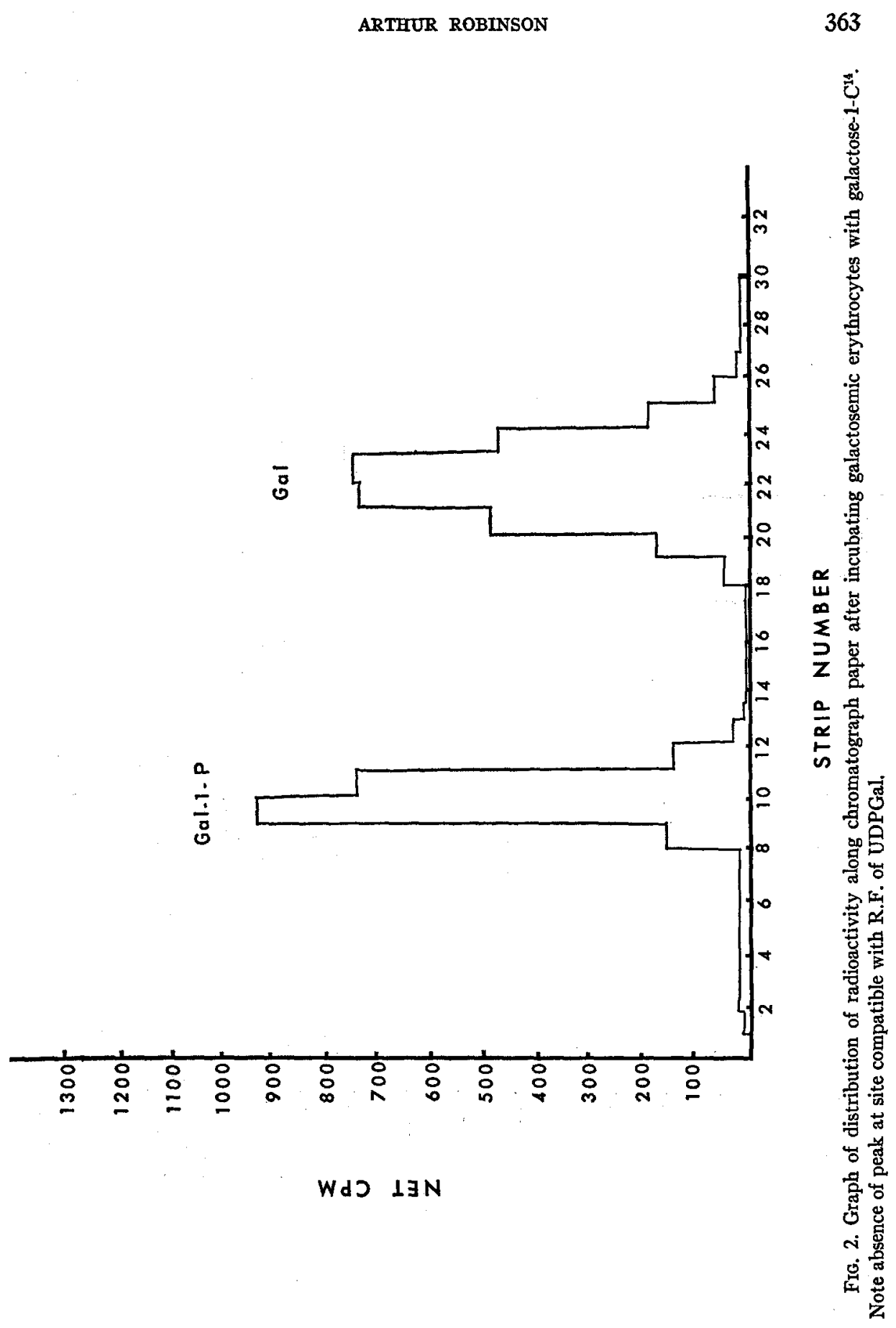




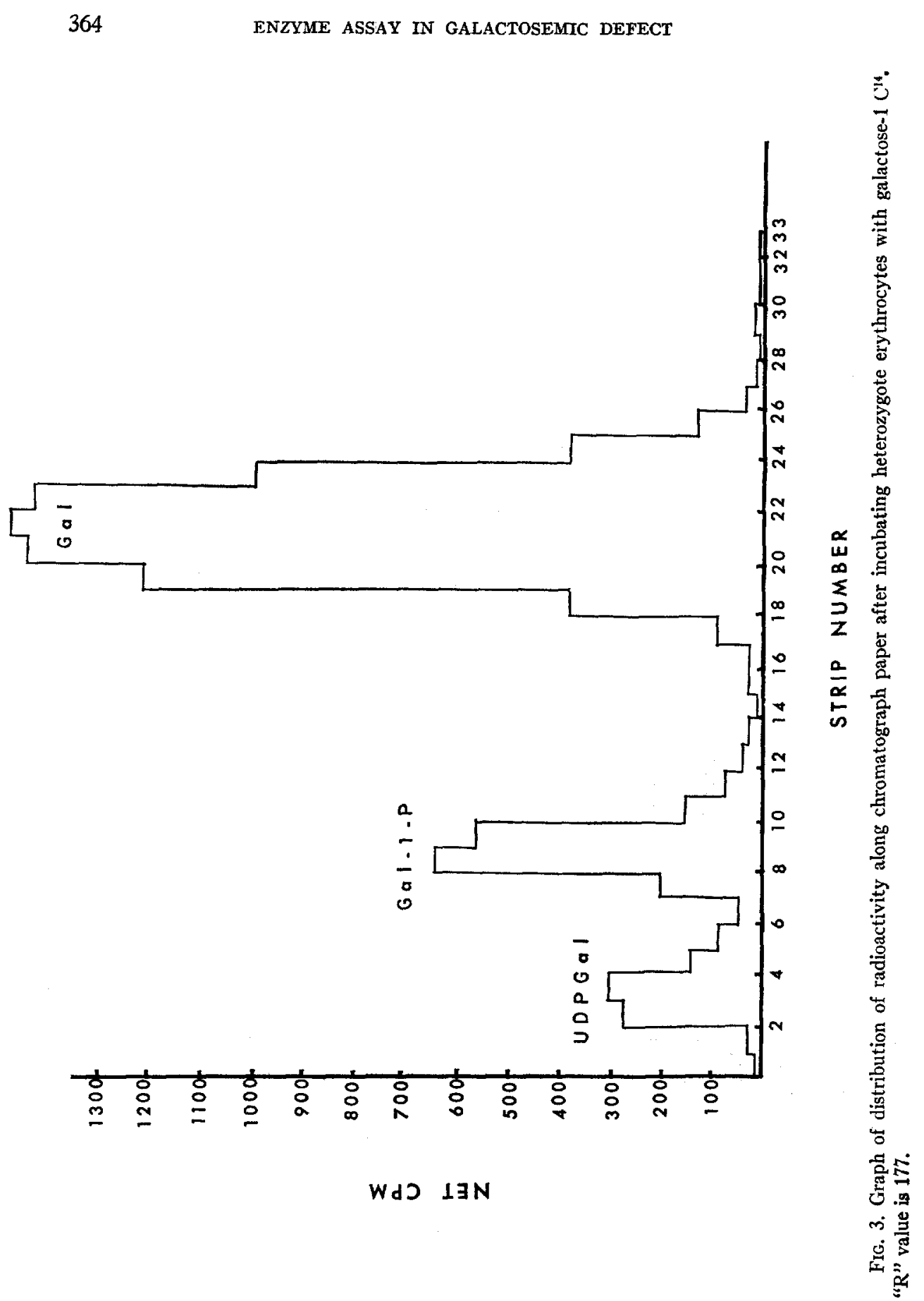


UDPGal was formed in any case (cf. Fig. 2). The sensitivity of the test was such that even an amount of UDPGal one-fiftieth as great as the Gal-1-phosphate produced could have been detected. Hence, the "R" value for all of these patients is at least $\mathbf{5 0 0 0 .}$

3. Results on Known Carriers of the Galactosemia Defect.--Six known carriers

TABLE I

" $R$ " Values of Erythrocytes of Healthy Individuals in the General Population (Controls)

\begin{tabular}{llll}
\hline 66 & 73 & 82 & \\
66 & 74 & 83 & \\
67 & 74 & 83 & \\
67 & 74 & 84 & \\
67 & 74 & 84 & Mean $=80$ \\
68 & 76 & 89 & sD $=12.4$ \\
68 & 76 & 92 & 96 per cent confidence limits \\
69 & 78 & 93 & $=55$ to 105 \\
72 & 78 & 94 & \\
73 & 81 & 99 & \\
73 & 81 & $103 *$ & \\
73 & 81 & $111 \ddagger$ & \\
& & $119 \ddagger$ &
\end{tabular}

* Subject was pregaant.

\$ These two determinations were done early in the procedures and were on individuals who could not be rechecked. No values on normal persons have since been that high.

TABLE II

Variability in One Individual

\begin{tabular}{|c|c|}
\hline 12 determinations & \\
\hline 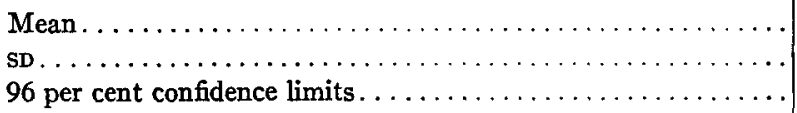 & $\begin{array}{c}74 \\
9 \\
56 \text { to } 92\end{array}$ \\
\hline
\end{tabular}

" $R$ " values of 12 determinations on erythrocytes performed on 6 different days.

of the recessive defect, i.e. the parents of the patients listed above, were available and tested by this procedure ( $c f$. Fig. 3). Values of the ratio are presented in Table III. This group reveals a mean value of " $R$ " of 170 , which is more than twice that of the normal population. The standard deviation of the group of known carriers is 20.5 , so that the significance of these results is obvious. This distinct difference between the values obtained from heterozygous carriers and members of the population at large suggests that the current procedure might serve as an unambiguous means for detection of heterozygous carriers.

4. Results on Siblings of Galactosemia Patients and on Relatives of Known 
Carriers.-As a final group of subjects, the siblings, cousins, and aunts of the galactosemic patients were also tested, in order to determine whether this test could reveal the presence of the recessive defect in the clinically normal persons of families where the genetic defect occurs. The data obtained (Table IV) reveal that the relatives are clearly divided into two distinct groups, one resembling the normal population, and the other resembling the known carriers in their response to this procedure. These results are made clear in Fig. 4, where all of the data from the three groups of disease-free subjects are analyzed. It is

TABLE III

" $R$ " Values for Erythrocytes of Carriers (Parents of Galactosemic Children)

\begin{tabular}{|c|c|c|}
\hline 1. Mr. B. & 155 & \\
\hline 2. Mrs. B........... & 162 & Mean $=170$ \\
\hline $3.1 \mathrm{Mr} .0 \ldots \ldots \ldots \ldots \ldots \ldots \ldots$ & 206 & $\mathrm{sD}=20.5$ \\
\hline 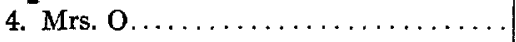 & 181 & 96 per cent confidence limits $=$ \\
\hline 5. Mr. W........... & 158 & 129 to 211 \\
\hline 6. Mrs. $W \ldots \ldots \ldots \ldots \ldots \ldots \ldots$ & 155 & \\
\hline
\end{tabular}

TABLE IV

" $R$ " Values of Erythrocytes of Relatives of Carriers

\begin{tabular}{l|c}
\hline 1. & 406 \\
2. & 167 \\
3. & 210 \\
4. & 222 \\
5. & 92 \\
6. & 70 \\
7. & 87 \\
8. & 78 \\
9. & 71 \\
\hline
\end{tabular}

particularly significant that in the total of 52 persons who have been examined, not 1 has yielded a result falling in the borderline region with " $R$ " values between 120 and 154, even when the 2 questionable, high values from Table I are included. The 9 relatives of the carriers are unequivocally identified by this procedure as to whether or not they possess a defective gene for the transferase enzyme. While these studies will be extended to larger groups, the data already available appear to offer promise of distinguishing carriers of this particular gene with a higher precision than has been hitherto possible.

5. Results on Cells in Tissue Culture.-Results on Hela cells and cells from skin of a patient with galactosemia grown in tissue culture reveal that the method can also be utilized on somatic cells in culture. Further studies with these are in progress. 


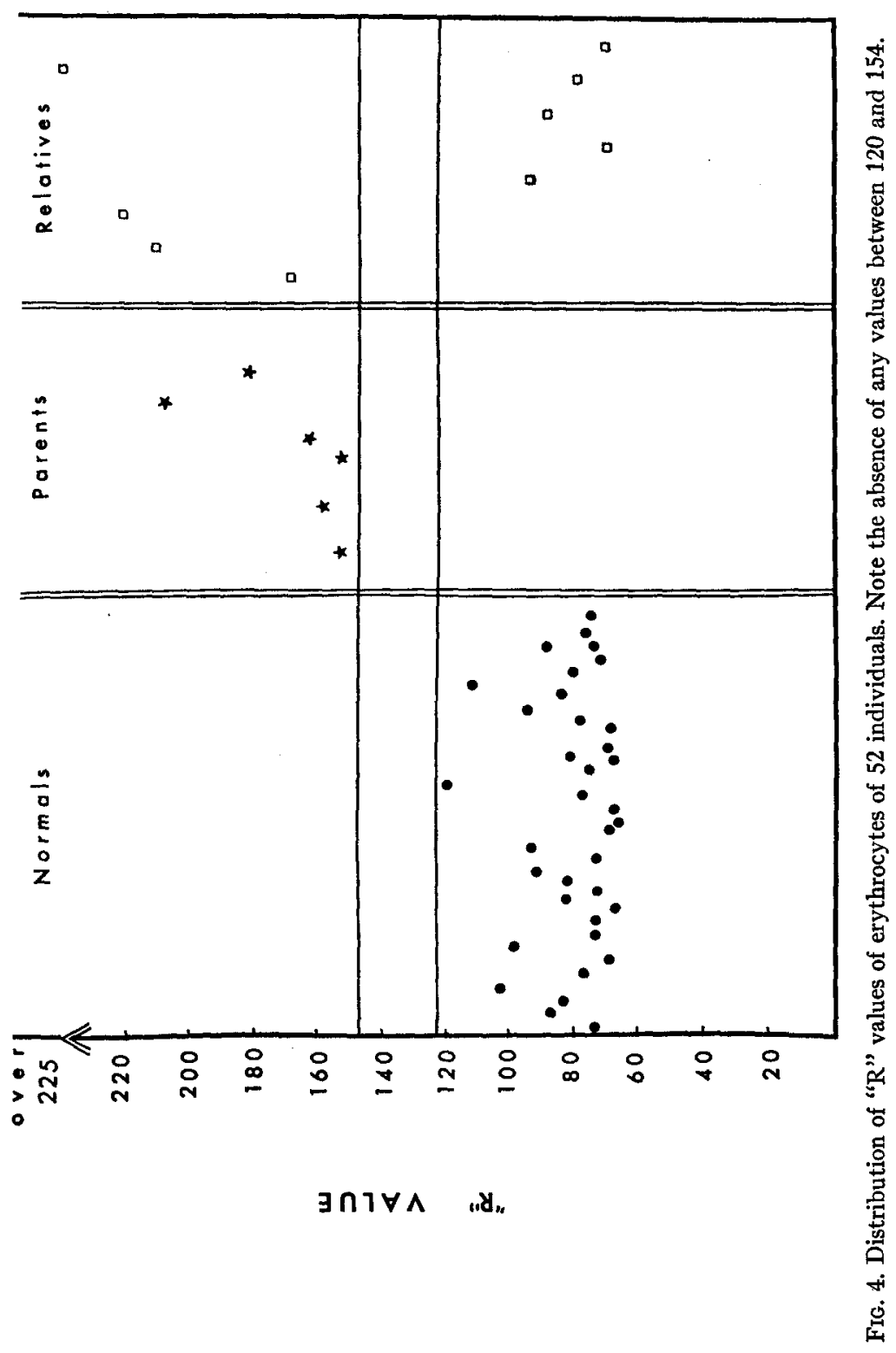




\section{DISCUSSION}

It is realized that in this solvent system it is impossible to differentiate the R.F.'s of the three galactose compounds from those of glucose, glucose-1phosphate, and uridine diphosphoglucose. Activity of the epimerase enzyme may, in fact, transform some of the labeled uridine diphosphogalactose to uridine diphosphoglucose. For this reason we have stated that the sites of radioactivity are compatible with those of the galactose compounds. While they might contain varying amounts of the glucose compounds also, these substances presumably also require integrity of $\operatorname{step} b$ in the metabolic sequence presented earlier, and hence would serve as well as UDPGal to indicate presence of a block. Thus the resulting ratios appear to characterize well the three groups of genotypes in relation to galactose metabolism. Studies of the detailed metabolic events in galactose metabolism are continuing.

Several methods have been developed for the determination of transferase enzymes in human erythrocytes. The three methods which to date have separated the three groups most distinctly are the UDPGlu consumption test (15-17); a test which measures the output of $\mathrm{C}^{14} \mathrm{O}_{2}$ of erythrocytes incubated with $\mathrm{C}^{14}$-labeled galactose (18); and a manometric assay (19) of the oxygen consumed in metabolism of a measured amount of galactose. In all of these, and particularly in the latter method, there is definite overlapping of the values obtained from the different groups of human subjects. In addition, the methods tend to be somewhat complex.

The method here described has the advantage of simplicity and reproducibility; lack of any demonstrated overlap between normals, heterozygous carriers, and homozygous persons with the disease; and direct measurement of the immediate reaction product of the blocked reaction $b$ so that galactose metabolism by other pathways is ruled out. Finally, the present method gives information on the presence of kinase as well as transferase enzyme.

This test offers the hope of identifying with reasonable reliability the heterozygous carriers in families of patients affected with galactosemia, and so is of value in genetic counseling. In addition, it furnishes a means for determining the frequency of the gene defect in a large population, without the serious assumptions implicit in the estimates based on the frequency of occurrence of the homozygous state in which the disease is present.

\section{SUMMARY}

A simple, rapid, and direct method for measuring the activities of the galactokinase and Gal-1-P uridyl transferase enzymes in human erythrocytes is presented.

The method has been applied to measurement of enzyme activities in a group of 37 presumably normal persons, 3 patients with galactosemia, 6 known 
heterozygous carriers of the defect, and 9 relatives of carriers. In every case the test unambiguously identified the individual's status as normal, heterozygotic carrier, or homozygously defective.

Use of the test in genetic counseling and genetic epidemological studies is discussed.

The author wishes to thank Dr. T. T. Puck and Dr. M. L. Morse for their helpful interest during this investigation.

The technical assistance of Evelyn Rice is gratefully acknowledged.

\section{BIBLIOGRAPHY}

1. Hugh-Jones, K., Newcomb, A. L., and Hsia, D. Y., The genetic mechanisms of galactosemia, Arch. Dis. Child., 1960, 35, 521.

2. Schwarz, V., Wells, A. R., Holzel, A., and Komrower, G. M., A study of the genetics of galactosemia, Ann. Human Gen., 1961, 25, 179.

3. Walker, F. A., Hsia, D. Y., Slatis, H. M., and Steinberg, A. G., Galactosemia: a study of twenty-seven kindreds in North America, Ann. Human Gen., 1962, $25,287$.

4. Kalckar, H. M., Anderson, E. P., and Isselbacher, K. J., Galactosemia, a congenital defect in a nucleotide transferase: a preliminary report, Proc. Nat. Acad. Sc., 1956, 42, 49.

5. Schwarz, V., Goldberg, L., Komrower, G. H., and Holzel, A., Some disturbances of erythrocyte metabolism in galactosemia, Biochem. $J$., 1956, 62, 34 .

6. Schwarz, V., The value of galactose phosphate determinations in the treatment of galactosemia, Arch. Dis. Child., 1960, 35, 428.

7. Leloir, L. F., The enzymatic transformation of uridine diphosphate glucose into a galactose derivative, Arch. Biochem. and Biophysics, 1951, 33, 186.

8. Kalckar, H. M., Braganca, B., and Munch-Peterson, A., Uridyl transferases and the formation of uridine diphosphogalactose, Nature, 1953, 172, 1038.

9. Leloir, L. F., The uridine coenzymes, Proc. Internat. Cong. Biochem., 3rd, Brussels, 1955, 1956, 154.

10. Isselbacher, K. J., A mammalian uridinediphosphate galactose pyrophosphorylase, J. Biol. Chem., 1958, 232, 429.

11. Morse, M. L., Lederberg, E. M., and Lederberg, J., Transduction in Escherichia coli K-12, Genetics, 1956, 41, 142.

12. Dormandy, T. L., and Porter, R. J., Familial fructose and galactose intolerance, Lancet, 1961, 1, 1189.

13. Maio, J. J., and Rickenberg, H. V., Metabolic block in utilization of galactose by strain L tissue culture cells, Science, 1961, 134, 1007.

14. Morse, M. L., personal communication.

15. Bretthauer, R. K., Hansen, R. G., Donnell, G., and Bergren, W. A., A procedure for detecting carriers of galactosemia, Proc. Nat. Acad. Sc., 1959, 45, 328.

16. Nordin, J. H., Bretthauer, R. K., and Hansen, R. G., Development and application of a simplified colorimetric method for detecting carriers of galactosemia, Clin. Chim. Acta, 1961, 6, 578. 
17. Bretthauer, R. K., Carbohydrate metabolism. I. Assessing heterozygosity for galactosemia by enzymatic means, A thesis submitted to Michigan State University, 1961.

18. Weinberg, A. N., Detection of congenital galactosemia and the carrier state using galactose $\mathrm{C}^{14}$ and blood cells, Metabolism, 1961, 10, 728.

19. Kirkman, H. N., and Bynum, E., Enzymic evidence of a galactosemic trait in parents of galactosemic children, Ann. Human Gen., 1959, 23, 117. 